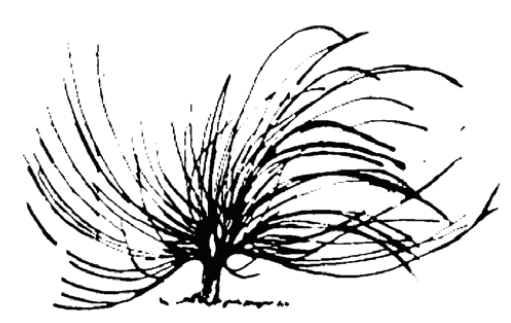

\title{
La Realidad del Uso de las TIC y su Mediación Pedagógica para Enriquecer las Clases de Inglés
}

\author{
Olga Chaves Carballo ${ }^{1}$ \\ Universidad Nacional \\ Heredia, Costa Rica \\ profeolgachaves@hotmail.com
Lindsay Chaves Fernández ${ }^{2}$
Universidad Nacional
Heredia, Costa Rica
lindsay.chaves.fernandez@una.cr \\ Didier Rojas Cerdas
Universidad Nacional
Heredia, Costa Rica
didierrojas@hotmail.com
}

\section{Resumen}

El objetivo principal de este artículo es identificar y analizar el uso de las TIC y la mediación pedagógica que los académicos del Área de Inglés de la Escuela de Literatura y Ciencias del Lenguaje (ELCL) utilizan para enriquecer las clases de inglés. A la luz del cumplimiento de los estándares de calidad de una carrera acreditada por el SINAES, los docentes del Área de Inglés reconocen la

\section{(c) (1) (\$) $\Theta$}

Recibido: 29 de junio de 2015-Aprobado: 26 de noviembre de 2015

1 Académica de la Escuela de Literatura y Ciencias del Lenguaje. Doctora en Educación con Énfasis en Mediación Pedagógica (Universidad de la Salle).

2 Académica de la Escuela de Literatura y Ciencias del Lenguaje. Master en Segundas Lenguas y Culturas con Énfasis en la Enseñanza del Inglés para Alumnado Adulto (Universidad Nacional).

3 Académico de la Escuela de Literatura y Ciencias del Lenguaje. Master en Segundas Lenguas y Culturas con Énfasis en la Enseñanza del Inglés para Alumnado Adulto (Universidad Nacional). 
importancia de mantenerse actualizados en las estrategias innovadoras con el apoyo de las TIC. Se ha evidenciado que estas promueven el aprendizaje mediante una gama de herramientas virtuales que potencian los estilos de aprendizaje, las inteligencias múltiples, y las necesidades especiales de la diversidad de aprendientes con el fin de mejorar su rendimiento académico. Este estudio se llevó a cabo en febrero del 2015 con una muestra de 27 docentes quienes han mostrado un esfuerzo e interés en ser conocedores de diferentes recursos tecnológicos que ellos utilizan en sus lecciones. Igualmente, los resultados del estudio indican que la ELCL se esmera por mejorar este ámbito ofreciendo capacitación docente más permanente.

Palabras clave: Tecnologías de la Información y la Comunicación (TIC), enseñanza del inglés, mediación pedagógica, formación docente

\begin{abstract}
The main objective of this paper is to identify and analyze the use of ICTs and the pedagogical mediation that professors of the English Department at the School of Literature and Language Sciences (ELCL) at Universidad Nacional (UNA) use to enhance their English classes. By complying with the quality standards of an accredited major by SINAES, professors of the English majors recognize the importance of being up-to-date when it comes to innovative strategies with the support of ICT. It is plausible that these state-of-the-art technologies promote learning through a series of virtual tools that foster the students' learning styles, multiple intelligences, and special needs, in order to improve their academic performance. This study was carried out in February, 2015 with a sample of 27 professors who have shown an effort and interest in knowing how to implement the different technological resources in their classes. Moreover, the results of this study demonstrate that the School of Literature and Language Sciences has gone to great lengths to improve in this area by offering constant professional development activities on a regular basis.
\end{abstract}


Keywords: Information and Communication Technologies (ICT), English teaching, pedagogical mediation, professional development

\section{Introducción}

Las Tecnologías de la Información y la Comunicación (TIC) contribuyen a la construcción de conocimiento en el sentido que existe una gran facilidad para acceder a más información, se incrementa las posibilidades de interactividad y conectividad, se utilizan más formatos multimedia. Es por lo tanto que la era de la tecnología y la comunicación exige de los docentes competencias en el uso de las TIC con el fin de mejorar el rendimiento estudiantil. Hoy en día, los académicos universitarios son conscientes de la necesidad de actualizar sus propias habilidades, y por consiguiente, se actualizan en el uso de las herramientas más exitosas en la enseñanza del inglés.

De esta manera, estas herramientas son el medio con el cual el docente de la enseñanza del inglés pueda utilizar la mediación pedagógica en los contenidos de un curso y así crear un ambiente de aprendizaje más dinámico, creativo, y real. Además, el docente que utiliza las TIC promueve el aprendizaje por medio de una gama de herramientas tecnológicas que desarrollan los estilos de aprendizaje, las inteligencias múltiples, y a las necesidades especiales de la diversidad de aprendientes.

En la Escuela de Literatura y Ciencias del Lenguaje (ELCL) se respira un ambiente de apropiación del uso de las TIC que les permite a los docentes mediar actividades y experiencias de aprendizaje significativas, más reales y dinámicas tanto en el salón de clase como fuera de él. Se ha motivado, capacitado y acompañado al académico para que se actualice en el uso de las TIC. Igualmente, el docente ha acogido muy dinámicamente la opción de utilizar las TIC como medios para promover el aprendizaje, compartir experiencias, reconstruir el conocimiento, reforzar las destrezas y micro destrezas del inglés, motivar extrínsecamente al estudiante, y maravillar sus mentes con contenidos novedosos. Por consiguiente, el objetivo principal de este artículo es identificar y analizar el uso de las TIC y la mediación pedagógica que los académicos del Área de Inglés de la Escuela de Literatura y Ciencias del Lenguaje (ELCL) utilizan para enriquecer las clases de inglés.

Debido a que el Bachillerato en la Enseñanza del Inglés (BEI) es una carrera acreditada por el Sistema Nacional de Acreditación de 
la Educación Superior (SINAES), la ELCL está comprometida con la excelencia por medio de procesos de autoevaluación y de acreditación. Una de las prioridades es la formación del docente en el uso de estrategias de enseñanza innovadoras. Debido a que las herramientas tecnológicas se han utilizado en la enseñanza de segundas lenguas exitosamente en las últimas dos décadas, los docentes del Área de Inglés de la ELCL utilizan las TIC con el fin de promover el aprendizaje y desarrollar las destrezas del lenguaje en espacios de aprendizaje más activos y dinámicos.

\section{Experiencias de aprendizaje en el mundo virtual}

En el nuevo paradigma tecnológico, las TIC han tomado un rol muy importante en la educación. Existe un universo de información, que no se puede compartir en la clase; por lo tanto, es imperante utilizar herramientas que les permitan a los estudiantes acceder a esta información por sus propios medios. Lo afirma Adams (2014) de la siguiente manera, "Las TIC con la adecuada mediación didáctica motivan a los estudiantes. El estudiante debe participar de una forma más activa así como también puede auto corregirse" (p.1).

Ávalos (2008) propone diferentes formas de implementar las TIC en el campo educativo que enriquezcan los procesos de enseñanza-aprendizaje, introduciendo estrategias novedosas para aprender a interactuar con la tecnología a partir de una adecuada planificación curricular. Algunas de ellas incluyen que los estudiantes se involucren en a) procesos de lecturas y análisis de texto, b) intervención en televisión interactiva, c) participación en actividades que les permitan leer, navegar e interactuar con el contenido propuesto, d) aportación en foros de discusión con el fin de promover el ambiente colaborativo, e) actividades que ofrezcan un aprendizaje significativo donde se les enfrente a situaciones desestructuradas, para tener la experiencia de organizarlas, f) participación de enlaces en subgrupos, entre otros.

Turkle (1997, p. 54) indica que "los individuos quieren relacionarse con la tecnología que les hace sentir cómodos y que refleja estilos personales." La nueva pedagogía de la educación en la era del conocimiento ha adoptado novedosos métodos de aprendizaje que le ayudan a los estudiantes a aprender en una manera holista e interactiva. 
Mediante el uso de las TIC exploramos un nuevo mundo de imágenes, de información con acceso inmediato para que el aprendiente pueda realizarse en la búsqueda del saber con su propio estilo de aprendizaje. De tal forma, lo recalca García-Vera (2004) "cuando interrogamos por el papel que juegan las nuevas tecnologías en la producción del saber, la respuesta es evidente: es el que siempre han jugado, uno muy importante, tanto que no es posible hablar de conocimiento sin referirse inmediatamente a la tecnología" (p. 35). Las nuevas tecnologías han llegado a ser parte de la vida de muchos aprendientes; en otras palabras, es prácticamente su mundo.

De forma similar, Gutiérrez (2009) hace una reflexión acerca de los retos que enfrenta la educación a partir del uso generalizado de las nuevas tecnologías. Si bien, los medios didácticos eran considerados como uno de los aspectos finales a tomar en consideración para el diseño instruccional del proceso educativo, este hecho evolucionó una vez que las tecnologías como soporte de transmisión de contenidos hacen posible el diseño de dichos medios didácticos. A partir de este acontecimiento se propicia entre los individuos, un modelo de interacción más participativo a través de medios tecnológicos. En general, estas nuevas herramientas ayudan a mediar los contenidos en una clase de inglés, facilitan el desarrollo de las destrezas y las habilidades cognitivas, analíticas y críticas.

\section{Metodología de la enseñanza y aprendizaje en el área de inglés}

Los avances en los marcos teórico-metodológicos de las ciencias, modifican también las teorías y metodologías para el estudio y abordaje de los procesos educativos donde se dimensiona su complejidad. Esta, a su vez, se expresa en los procesos educativos en el aula como la necesidad de atender en el espacio multicultural que aquella representa, las diversas formas de inteligencia para propiciar su desarrollo, la utilización de tecnologías y metodologías para la enseñanza y el aprendizaje, en concordancia con esta micro cultura, así como la promoción y construcción de prácticas de principios éticos.

En el año 2013 las académicas Pizarro y Cordero (2013) realizaron un estudio con académicos de las tres áreas: inglés, francés y español en la ELCL y concluyen que el uso de las TIC mejora la calidad 
de la enseñanza y rompe barreras de tiempo y espacio, "son muchos los beneficios que las tecnologías de información y comunicación (TIC) ofrecen en los contextos de educación superior" (p. 278). Este estudio refleja el reconocimiento, por parte del profesorado, de que las TIC ofrecen material complementario actualizado, el cual permite que las clases sean más innovadoras.

En elaño2012 Ramírez, Cañedoy Clemente estudiaron las actitudes $\mathrm{y}$ creencias que los profesores de secundaria tienen sobre la utilización de los recursos de Internet en sus prácticas. Entre los datos más significativos, el estudio demuestra que el $92.1 \%$ de los profesores consideran que el Internet es un recurso formativo para los alumnos.

Díaz Larenas, Jansson Bruce y Neira Martínez (2011) en su estudio indican que la tecnología es un aporte para la enseñanza y aprendizaje de la lengua, ya que promueve aprendizajes significativos. Estos autores enfatizan que la tecnología permite una re-distribución de los roles de profesor y estudiante, aumenta la motivación y mejora el aprendizaje; sin embargo, su estudio reveló que su uso se limita a la sala de clases y que aún no es aprovechada en todo su potencial, ya que se subutilizan aplicaciones y recursos que podrían mejorar aún más los aprendizajes de los estudiantes.

Es precisamente en esa línea de pensamiento que se inscribe la necesidad de fortalecer y profundizar la formación docente, desde un currículum interdisciplinario y con el apoyo de la virtualidad, lo cual significa que la formación del docente se enriquece desde diversos saberes, para que comprenda mejor los procesos educativos y sus contextos particulares e intervenga en ellos y facilite la construcción de opciones y soluciones a los retos que enfrente cotidianamente en el aula.

Es un hecho que los docentes deseamos eficiencia en el proceso de mediación pedagógica; cada año, nuestras aulas se van renovando con estudiantes de generaciones más recientes. Tal es el caso que en el año 2018 recibiremos los primeros estudiantes nacidos en el siglo XXI. Paradójicamente, los docentes vamos avanzando en edad. Sin embargo, esto no debe ser negativo, lo que sí debemos entender es que estamos obligados a actualizarnos. Al respecto, Chaves y Gutiérrez (2007, p. 37) argumentan,

Cuando el proceso de aprendizaje se realiza a través de medios digitales, debe existir un tutor, con funciones de facilitador, 
asesor, consultor y motivador del conocimiento, cuya interacción con el aprendiente sea para compartir con él sus experiencias, apoyarlo y asesorarlo en su proceso de aprender, estimulándole su capacidad de adquirir conocimiento, retándolo a incrementar sus saberes, promoviendo la creación de su propio paradigma, en donde se pueda apropiar y adueñar de sus conocimientos, para luego compartirlos con los demás y así crecer, bajo una interacción presencial y virtual.

No es una opción quedarnos estancados en los métodos y tecnologías que usábamos en el pasado. Seremos mucho más efectivos cuando aprendamos los lenguajes de las nuevas generaciones. En un estudio hecho por Fernández, Server y Carballo (2006), se evidencia que, con la incorporación de las TIC en la enseñanza, “... el alumno se transforma en participante activo y constructor de su propio aprendizaje y el profesor asume el rol de guía y facilitador. . lo cual varía su forma de interactuar con sus alumnos, la forma de planificar y de diseñar el ambiente de aprendizaje" (p. 1).

Barrera (2010, p. 18) cita a la Secretaría de Educación Pública para enfatizar las ventajas que se obtienen al adquirir una formación continua y desarrollarse en el uso y conocimientos en tecnologías de la comunicación.

...la formación continua como el conjunto de actividades que permiten a un docente desarrollar nuevos conocimientos y capacidades a lo largo de su ejercicio profesional y perfeccionarse después de su formación inicial. La formación continua del profesor de cualquier nivel educativo consiste en la actualización y capacitación cultural, humanística, pedagógica y científica con el fin de mejorar permanentemente su actividad profesional.

Los académicos del Área de Inglés de la ELCL se han comprometido a recibir capacitación permanente en los últimos cinco años como lo indica la tabla 1. 
Tabla 1. Capacitaciones en la ELCL entre 2010 y 2015

\begin{tabular}{|c|c|c|}
\hline Año & Curso o Taller & $\begin{array}{c}\text { Instancia } \\
\text { Facilitadora }\end{array}$ \\
\hline \multirow[t]{2}{*}{2010} & Sitio Web del Académico & UNA \\
\hline & Congreso Internacional de Educación Superior & UNA \\
\hline \multirow[t]{2}{*}{2011} & $\begin{array}{l}\text { III Congreso Internacional de Lingüística } \\
\text { Aplicada }\end{array}$ & UNA \\
\hline & Evaluación y medición de los aprendizajes & MEP-CONARE \\
\hline $\begin{array}{l}2011- \\
2012\end{array}$ & $\begin{array}{l}\text { Diseño de programas, proyectos y actividades } \\
\text { académicas en el marco de lingüística aplicada a } \\
\text { la enseñanza del inglés }\end{array}$ & PEADP \\
\hline \multirow[t]{5}{*}{2012} & $\begin{array}{l}\text { Exploring Web 2.0: Tools for Classroom } \\
\text { Teaching and Professional Development. }\end{array}$ & $\begin{array}{l}\text { Online; Lewis \& } \\
\text { Clark College, } \\
\text { Oregon }\end{array}$ \\
\hline & $\begin{array}{l}\text { Fortalecimiento de habilidades curriculares y } \\
\text { tecnológicas en la enseñanza del inglés }\end{array}$ & $\mathrm{DE}$ \\
\hline & How to Design Integrated Skills Tests? & ELCL \\
\hline & $\begin{array}{l}\text { Estrategias para la redacción de artículos } \\
\text { académicos }\end{array}$ & ELCL \\
\hline & $\begin{array}{l}\text { Diseño de cursos, estrategias didácticas y } \\
\text { evaluación con enfoque en competencias }\end{array}$ & $\begin{array}{l}\text { Esc. } \\
\text { Bibliotecología }\end{array}$ \\
\hline $\begin{array}{l}2012- \\
2013\end{array}$ & $\begin{array}{l}\text { Formación docente para la innovación } \\
\text { pedagógica con TIC }\end{array}$ & ELCL \\
\hline \multirow[t]{6}{*}{2013} & $\begin{array}{l}\text { Charla sobre los recursos bibliográficos y bases } \\
\text { de datos }\end{array}$ & ELCL \\
\hline & $\begin{array}{l}\text { IV Congreso Internacional de Lingüística } \\
\text { Aplicada }\end{array}$ & ELCL \\
\hline & $\begin{array}{l}\text { Redacción y argumentación para docentes } \\
\text { universitarios }\end{array}$ & ELCL \\
\hline & Microsoft Office Excel 2007 & CATIA \\
\hline & $\begin{array}{l}\text { Utilización del formato APA en producción } \\
\text { académica }\end{array}$ & ELCL \\
\hline & Uso del lenguaje en la producción académica & ELCL \\
\hline
\end{tabular}




\begin{tabular}{|c|c|c|}
\hline \multirow[t]{11}{*}{2014} & II Simposio Impacto de las TIC en la educación & CIDE, UNA \\
\hline & $\begin{array}{l}\text { El inter-aprendizaje en la educación virtual: } \\
\text { propuestas para dialogar y repensar las } \\
\text { estrategias pedagógicas }\end{array}$ & UNA \\
\hline & Taller edición de video & UNA \\
\hline & Taller Prezi & ELCL \\
\hline & $\begin{array}{l}\text { IV Congreso Internacional de Educación } \\
\text { Superior (CIESUP) }\end{array}$ & $\begin{array}{l}\text { UNA y West } \\
\text { Chester University, } \\
\text { Penn, USA }\end{array}$ \\
\hline & $\begin{array}{l}\text { Estrategias de aprendizaje para estudiantes con } \\
\text { discapacidad visual }\end{array}$ & PEADP \\
\hline & $\begin{array}{l}\text { Desarrollo de habilidades pedagógicas y } \\
\text { técnicas en el uso de las pizarras digitales } \\
\text { interactivas }\end{array}$ & PEADP \\
\hline & $\begin{array}{l}\text { Implementación de un curso universitario } \\
\text { presencial con apoyo tecnológico }\end{array}$ & PEADP \\
\hline & $\begin{array}{l}\text { How Can We Blend Learning, Combine } \\
\text { Additional Oral Production and still Find Time } \\
\text { to Cover our English Syllabus? }\end{array}$ & ELCL \\
\hline & Sistematización de experiencias de aula & ELCL \\
\hline & Jornadas Académicas & UNA \\
\hline \multirow[t]{7}{*}{2015} & $\begin{array}{l}\text { Desarrollo de habilidades pedagógicas y } \\
\text { técnicas en el uso de las pizarras digitales } \\
\text { interactivas }\end{array}$ & PEADP \\
\hline & Coop Learning Workshop & ELCL \\
\hline & $\begin{array}{l}\text { Curso: Métodos y técnicas para la Investigación } \\
\text { en ciencias de la educación y del lenguaje }\end{array}$ & ELCL \\
\hline & IV Foro de Académicos Innovadores con TIC & $\begin{array}{l}\text { Programa UNA } \\
\text { Virtual }\end{array}$ \\
\hline & $\begin{array}{l}\text { III Congreso Internacional de Investigación } \\
\text { Educativa: Globalización y Educación }\end{array}$ & UCR \\
\hline & $\begin{array}{l}\text { Curso: Fortalecimiento de las destrezas } \\
\text { investigativas }\end{array}$ & ELCL \\
\hline & $\begin{array}{l}\text { Taller sobre el manejo de Base de Datos del } \\
\text { SIDUNA }\end{array}$ & CIDE \\
\hline
\end{tabular}

Nota: Información tomada del Compromiso de Mejoramiento del BEI elaborada por los investigadores 
Además, en la encuesta realizada a los 27 académicos se incluyen capacitaciones en CONARE, MEP, UCR, TEC y asociaciones profesionales. La tabla 1 indica que en los últimos dos años, los académicos han efectivamente desarrollo más destrezas tecnológicas debido a las demandas educativas en el campo de la enseñanza del inglés. Además, los docentes reconocen que los estudiantes de este milenio utilizan las TIC con facilidad y dominio para fortalecer sus destrezas lingüísticas. Igualmente, los estudios de investigación muestran que las TIC promueven el aprendizaje y que los académicos en la actualidad están conscientes de sus ventajas en el campo de la educación. Lo más importante es reconocer que debemos continuar formándonos para apropiarnos de su uso por medio de la mediación pedagógica exitosa.

\section{Metodología del estudio}

Para este estudio se realizó una investigación descriptiva de campo con un enfoque cualitativo interpretativo. El estudio se llevó a cabo en febrero del 2015 en un contexto real con la participación de 27 académicos del Área de Inglés de la ELCL, los cuales contestaron un cuestionario de 11 preguntas, tanto abiertas como cerradas, concernientes al uso de las TIC en el salón de clase y la capacitación con la que cuentan para utilizar estas tecnologías en la práctica en su quehacer docente. El cuestionario fue elaborado por los investigadores, considerando que tienen amplia experiencia en previos estudios realizados en el Área de Inglés a raíz de los procesos de Autoevaluación, Acreditación y Re acreditación de la Carrera del BEI. La acreditación fue otorgada por el SINAES en el año 2006 y ahora cuenta con la Reacreditación hasta el año 2017.

Durante el diseño del instrumento utilizado para este estudio participaron tres miembros de la Comisión de Mejoramiento, junto con el asesoramiento de un experto del Programa de Diseño y Gestión Curricular. Para esta ocasión, se utilizó un formulario en línea de Google Drive y se envió a los académicos por correo electrónico, ya que se ha implementado en la ELCL, el hábito de realizar este tipo de cuestionarios en línea. El cuestionario se encuentra en la siguiente dirección: http://goo. gl/forms/g2ZKqJG5WA (Ver versión impresa en el Apéndice). 


\section{Resultados y discusión}

Los resultados de la encuesta (preguntas 1 y 2) revelan que los académicos del Área de Inglés utilizan una gran variedad de herramientas tecnológicas y recursos digitales disponibles en Internet, ya que han aprendido a utilizarlos por medio de diversas capacitaciones de interés académico y personal. Se evidencia, por lo tanto, que se utilizan aparatos electrónicos aún tradicionales como los proyectores, grabadora y laboratorios de idiomas hasta lo más moderno como los celulares inteligentes. Estos medios digitales tradicionales son indispensables para enseñar un segundo idioma debido a la gran cantidad de material didáctico que se puede utilizar y desarrollar en la mediación pedagógica para alcanzar los objetivos de los diversos cursos de la carrera.

Igualmente, el correo electrónico, los foros en el aula virtual, Skype y los wikis permiten la comunicación constante y la interacción profesor-estudiante en la mediación de actividades de clase, tareas y proyectos colaborativos. Para dar presentaciones orales, se ha implementado el uso del Prezi cuyo uso fortalece el estilo de aprendizaje visual, la creatividad y la expresión oral de los estudiantes debido a su dinamismo y novedad. El uso de videos en Youtube es esencial en una clase de idiomas porque presenta la realidad, son interactivos y se pueden utilizar en todo momento. Otras aplicaciones web más innovadoras e interactivas son los wikis, el Voicethread, el Voki, el Spiderscribe, y los blogs con las cuales se pueden mediar un sinnúmero de actividades como grabaciones de diálogos y descripciones, práctica de pronunciación, redacciones, actividades con vocabulario, y creación de esquemas, entre otras tareas, para facilitar el aprendizaje de temas y desarrollar las funciones en el inglés.

Además de los usos anteriores, los académicos utilizan las diferentes TIC en varios escenarios en sus clases de idioma. Ellos argumentan que debido a lo actualizado de la información que encuentran en la web, ésta es en definitiva la más indicada para desarrollar tanto la práctica oral como escrita en los estudiantes. Esto lo llevan a cabo de distintas maneras:

1. Lectura y discusión de artículos encontrados en la web

2. Comprensión de lectura al contestar preguntas referentes a una lectura 
3. Comprensión auditiva al contestar preguntas referentes a un video

4. Discusión de preguntas generadoras después de ver un video

5. Escritura de un ensayo para analizar una lectura o un video.

Además, esta información les es útil para complementar otros materiales de índole tradicional que se utilizan en clase como los libros de texto y antologías. De esta manera, la práctica de temas gramaticales, por ejemplo, se puede realizar de una manera más interactiva utilizando páginas web y CD ROM.

Otro punto valioso en cuanto al uso de las TIC en educación superior, según los académicos de la ELCL, es la exposición a nativos parlantes del inglés que pueden tener los estudiantes sin necesidad de salir del país, lo cual repercute de manera positiva en la adquisición de una lengua extranjera. Esto a su vez, se relaciona con el conocimiento cultural que pueden adquirir los alumnos mediante vídeos, lecturas, redes sociales, blogs, tutoriales y documentales, los cuales son utilizados tanto por los académicos para realizar su mediación pedagógica en el aula, como por los mismos estudiantes en sus horas de estudio independiente para afianzar sus conocimientos en el idioma y cultura metas. En este punto, cabe resaltar que los profesores utilizan vídeos para la comprensión auditiva y la mejora de la pronunciación.

Otro medio tecnológico que se encuentra en auge en la ELCL es el uso del Aula Virtual Institucional, ya que su uso permite a los docentes un acercamiento a sus estudiantes fuera de las horas de clase presencial, y ésta se puede utilizar en conjunto con otras herramientas virtuales como lo son el Voicethread, Voki, podcasts y enlaces a distintas páginas web. Debido a su gran versatilidad, el uso del Aula Virtual como mediación pedagógica también se extiende a las lecciones presenciales para apoyar las actividades que trae el libro de texto.

El uso de los celulares inteligentes de los estudiantes también se ha vuelto una práctica común en las clases de inglés de la ELCL, ya que éstos permiten que los estudiantes realicen una serie de actividades que van en incremento de la adquisición de la lengua meta. Por ejemplo, algunas de las acciones que se pueden realizar con el uso de celulares son las siguientes:

1. Grabaciones de los estudiantes para autocorregir su pronunciación o la de un compañero 
2. Acceso al Aula Virtual

3. Acceso a páginas web para realizar prácticas durante la clase

4. Grabaciones de video

5. Acceso a diccionarios en línea que contienen tanto las definiciones como la pronunciación por un nativo parlante de la palabra en cuestión

6. Acceso a información para investigación

Las redes sociales también juegan un papel muy importante en la mediación pedagógica que realizan los académicos, ya que mediante éstas, los profesores pueden tener acceso inmediato a sus estudiantes para brindarles toda aquella información que ellos consideren necesaria.

No cabe duda que al utilizar estas herramientas tecnológicas se desarrollan todas las destrezas lingüísticas, se incrementa el vocabulario y se mejora la pronunciación. Asimismo, los profesores consideran muy valioso el hecho de que al utilizar tecnología en sus clases, promueven en sus alumnos su uso futuro, cuando impartan sus propias lecciones.

\section{Tabla 2. Porcentaje de académicos que utilizan los siguientes recursos tecnológicos}

\begin{tabular}{|l|l|l|l|}
\hline \multicolumn{1}{|c|}{$\mathbf{1 0 0 \% - 8 0 \%}$} & \multicolumn{1}{|c|}{$\mathbf{8 0 \% - 6 0 \%}$} & \multicolumn{1}{|c|}{$\mathbf{6 0 \% - 4 0 \%}$} & \multicolumn{1}{|c|}{$\mathbf{4 0 \% - 2 0 \%}$} \\
\hline Computadoras & Celulares & Softwares & Cámara \\
Proyectores & inteligentes & especiales para & fotográfica y de \\
Office & Tabletas & la enseñanza del & video \\
Laboratorio de idiomas & Redes sociales & inglés & Educaplay \\
Grabadora & Skype & Pizarra & Blogs \\
Fórums & Windows movie & electrónica & Mindmeister \\
Páginas web & maker & Podcasts & Powtoon \\
Correo electrónico & Wikis & Chat & Herramientas \\
DVD & & Voicethread & para traducción \\
Prezi & & Voki & asistida \\
Youtube & & Spiderscribe & Google books \\
Aula virtual & & & Diigo \\
Diccionarios en línea & & & \\
Dropbox & & & \\
Google drive & & & \\
\hline
\end{tabular}

Nota: Información tomada de cuestionario en línea elaborado por los investigadores. 
Mediante las respuestas obtenidas en la pregunta 3 es posible identificar que una gran mayoría de académicos, $60 \%$, utilizan las TIC en ambas sesiones (en un curso que se imparte dos veces por semana) y un $20 \%$ en una sesión por semana. Este resultado indica que los académicos muy a menudo hacen uso de las herramientas virtuales para impartir sus lecciones. Esto es sin duda, una estadística alentadora debido a todos los usos innovadores y dinámicos que los académicos le están dando a las TIC en sus clases, lo cual, incide positivamente en la adquisición de la lengua meta por parte de los estudiantes. Además, en la pregunta 4 , los profesores hacen evidente que los recursos que proporciona la web forman parte de sus planeamientos y, por consiguiente, de sus lecciones, ya que un $63 \%$ indican siempre utilizarlos y un $37 \%$ lo hacen en algunas ocasiones.

Los académicos, consideran importante el material que se encuentra en la web (pregunta 5) porque tal y como ellos apuntan, el recurso que ofrece la web es novedoso, dinámico, fácil de utilizar, gratuito, eficaz, y expedito; además, es fácil para compartir con los estudiantes y para fortalecer el aprendizaje independiente. Asimismo, los docentes opinan que es necesario utilizar este material para estar actualizado, satisfacer necesidades especiales de los estudiantes, complementar y adaptar otros materiales ya existentes. También señalan, como se expuso anteriormente en este escrito, que desean que al utilizarlos, sus estudiantes estén preparados para hacerlo cuando sean docentes con sus propios estudiantes. Las TIC definitivamente, brindan una oportunidad para exponer al estudiante a hablantes nativos del idioma y para desarrollar actividades de investigación. Esto demuestra el nuevo paradigma del docente de acuerdo con Blanco (s. f., p. 2).

El profesor deja su faceta de experto en contenidos, presentador y transmisor de información y se convierte fundamentalmente en un diseñador de medios, un facilitador del aprendizaje y un orientador del estudiante, lo que supondrá que realice diferentes tareas como son: diseñar actividades de aprendizaje y evaluación, ofrecer una estructura para que los alumnos interaccionen, o animar a los estudiantes hacia el autoaprendizaje.

Como se puede observar mediante lo expuesto en este artículo, la mediación pedagógica que los académicos han implementado en el aula 
de inglés es variada y dinámica; se utilizan actividades o tareas para desarrollar todas las habilidades, para formar al aprendiente, para crear hábitos de estudio y para mantener una comunicación permanente.

La pregunta 6 , brinda un panorama de las destrezas del inglés que los académicos buscan desarrollar con el uso de las diferentes herramientas tecnológicas.

Tabla 3. Destrezas desarrolladas mediante el uso de las TIC

\begin{tabular}{|c|c|c|c|c|}
\hline TIC utilizada & \begin{tabular}{|c|}
$\begin{array}{c}\text { Comprensión } \\
\text { auditiva }\end{array}$ \\
\end{tabular} & \begin{tabular}{|c|c|} 
Comprensión de \\
lectura
\end{tabular} & \begin{tabular}{|c|}
$\begin{array}{c}\text { Comunicación } \\
\text { oral }\end{array}$ \\
\end{tabular} & Escritura \\
\hline Youtube & $\mathrm{x}$ & $X$ & $\mathrm{x}$ & $\mathrm{x}$ \\
\hline Prezi/Office & & $\mathrm{X}$ & $\mathrm{x}$ & $\mathrm{x}$ \\
\hline $\begin{array}{l}\text { Laboratorio de } \\
\text { idiomas }\end{array}$ & $\mathrm{x}$ & $X$ & $\mathrm{x}$ & $\mathrm{x}$ \\
\hline $\begin{array}{l}\text { Softwares } \\
\text { especiales }\end{array}$ & & & $\mathrm{x}$ & $\mathrm{x}$ \\
\hline Aula virtual & $\mathrm{x}$ & $\mathrm{x}$ & $\mathrm{x}$ & $\mathrm{x}$ \\
\hline Voicethread & $\mathrm{x}$ & $\mathrm{x}$ & $\mathrm{x}$ & $\mathrm{x}$ \\
\hline Voki & $\mathrm{x}$ & & $\mathrm{x}$ & \\
\hline Spiderscribe & & $\mathrm{x}$ & & $\mathrm{x}$ \\
\hline Skype & $X$ & & $\mathrm{x}$ & \\
\hline $\begin{array}{l}\text { Dropbox/Google } \\
\text { Drive/Correo } \\
\text { electrónico }\end{array}$ & $X$ & $\mathrm{x}$ & & $\mathrm{x}$ \\
\hline $\begin{array}{l}\text { Celulares } \\
\text { inteligentes/ } \\
\text { Tabletas }\end{array}$ & $X$ & & $\mathrm{x}$ & \\
\hline Podcasts & $\mathrm{X}$ & & $\mathrm{x}$ & \\
\hline Wikis & $\mathrm{X}$ & $\mathrm{x}$ & & $\mathrm{x}$ \\
\hline Blogs/Fórums & & $\mathrm{x}$ & & $\mathrm{x}$ \\
\hline Libros virtuales & & $\mathrm{x}$ & & \\
\hline Redes socials & $\mathrm{X}$ & $\mathrm{x}$ & & $\mathrm{x}$ \\
\hline Páginas web & $X$ & $\mathrm{x}$ & $\mathrm{x}$ & $\mathrm{x}$ \\
\hline DVDs, grabadoras & $X$ & & $\mathrm{x}$ & $\mathrm{x}$ \\
\hline
\end{tabular}

Nota: Información tomada de cuestionario en línea elaborado por los investigadores. 
La tabla 3 indica que los docentes pueden mediar algunas destrezas lingüísticas del inglés por medio de una serie de estas herramientas tecnológicas. Una de esas destrezas es la verbal y de ese modo, los estudiantes lograrán sus objetivos académicos.

Además de las destrezas arriba mencionadas, los docentes consideran que con el uso de las TIC también es posible ayudar a los estudiantes a mejorar su gramática y pronunciación.

\section{Figura 1. Aspectos que los profesores buscan fomentar con el uso de las TIC}

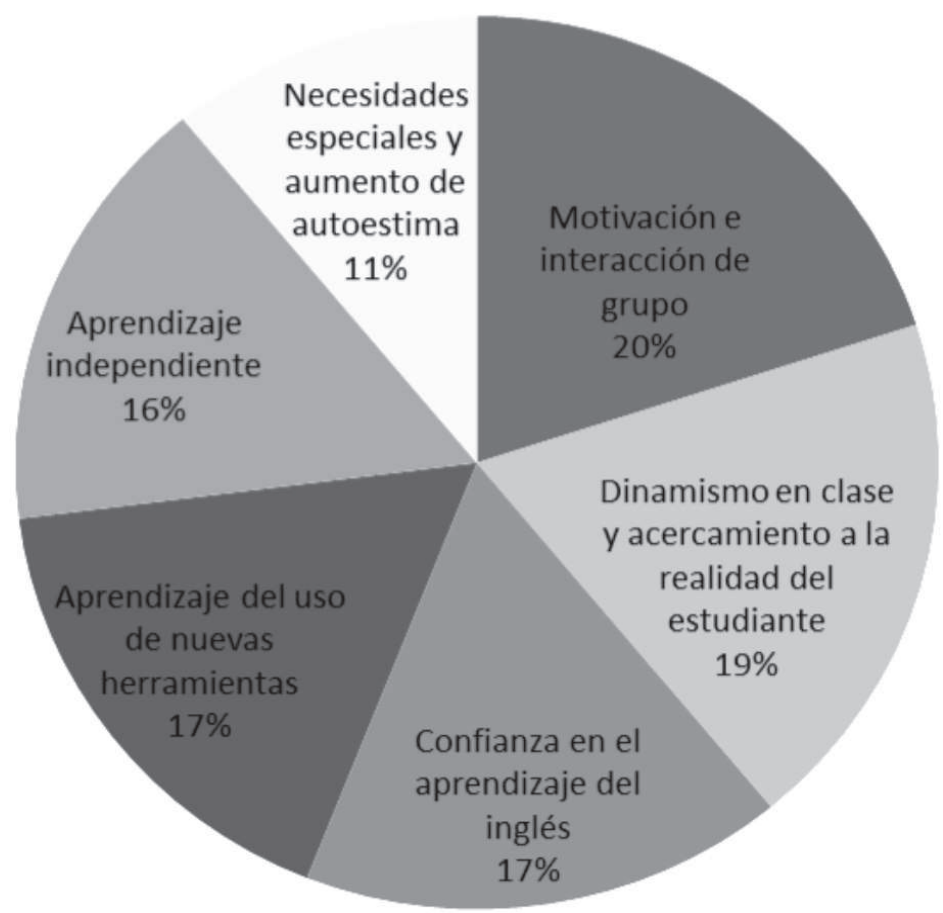

Nota: Información tomada de cuestionario en línea elaborado por los investigadores

Igualmente y de acuerdo con las respuestas brindadas por los profesores, el uso de las TIC promueve el trabajo en grupo, la tolerancia e interés hacia otras culturas, pensamiento crítico, humanismo, desarrollo de habilidades de investigación, destrezas académicas y profesionales, inteligencias múltiples, creatividad, descubrimiento de otros intereses y habilidades de los estudiantes, rapport (empatía) entre estudiante y profesor, respeto entre estudiantes, apoyo para estudiantes con nivel más bajo, aprovechamiento de la información reciente, reto al utilizar herramientas más avanzadas, ahorro de papel, entre otras. 
Finalmente, unos pocos académicos indican que les gustaría recibir aún más capacitación para sentirse más preparados y empoderados en el uso de pizarras interactivas, blogs, Excel, Google drive, Dropbox, Prezi y aula virtual. Esta actitud revela que existe un sentir de innovación educativa muy arraigado por parte de los académicos de la ELCL quienes reconocen el boom educativo de las TIC en la enseñanza de segundas lenguas.

El estudio, en general, revela que en efecto los académicos del Área de Inglés utilizan las TIC para enriquecer sus clases. Se da énfasis en las capacitaciones que han llevado para poder visualizar el tipo de herramienta o aplicaciones que conocen. Sin embargo, el estudio se limitó a identificar y analizar el uso de las TIC. Aún no se analiza el efecto cualitativo que tienen las mismas en el mejoramiento de las destrezas lingüísticas de los discentes. Por lo consiguiente, un estudio futuro medirá el impacto que tiene el uso de las TIC en las competencias lingüística de los estudiantes.

\section{Recomendaciones}

1. Continuar ofreciendo a los docentes formación en el uso de las TIC que les facilite la búsqueda de prácticas innovadoras para su quehacer académico.

2. Desarrollar en la academia una actitud abierta hacia las nuevas tendencias tecnológicas que van haciendo su aparición en la vida cotidiana de cualquier estudiante.

3. Formar una comunidad profesional de aprendizaje, "professional learning communities", tal como lo recomienda Nussbau y Ritter (2012, p. 29). la cual consiste en estar permanentemente conectados en equipos colaborativos para apoyarse, alcanzar metas y continuar aprendiendo en línea acerca de las TIC. Estos autores comentan, "colleagues commonly visit classrooms to offer feedback, and members share a vision and support the group's activities".

4. Además, buscar la adaptación y flexibilidad mental. Como menciona Beltrán $(2014$, p.1) "flexibilidad mental es tener una mente adaptable, tiene mucho que ver con soltar el control, dejar de aferrarnos a nuestras creencias de lo que es correcto o incorrecto". 
5. Para futuras investigaciones acerca de este tema, se debe medir el mejoramiento lingüístico en los discentes al estar expuestos a cada una de las herramientas que este estudio ha identificado.

6. Igualmente, se debe estudiar de qué medida cada capacitación contribuye cualitativamente en la mediación pedagógica que el docente diseña en sus lecciones para fortalecer diferentes destrezas lingüísticas.

\section{Conclusión}

El objetivo principal de este estudio fue identificar y analizar el uso de las TIC y la mediación pedagógica que los académicos del Área de Inglés de la Escuela de Literatura y Ciencias del Lenguaje (ELCL) utilizan para enriquecer las clases de inglés. El instrumento utilizado cumplió con el objetivo, ya que nos arrojó datos bastante amplios acerca de los tipos de herramientas tecnológicas que los docentes usan y también, la frecuencia con la que las utilizan en sus clases. En ambos apartados, los números fueron amplios, es decir, que los docentes son usuarios altamente activos y con una alta variedad de recursos tecnológicos. Por otro lado, el estudio deja ver que los docentes usan las TIC con un propósito bastante claro; entre esas razones tenemos el promover el trabajo en grupo, la tolerancia e interés hacia otras culturas, el pensamiento crítico, el humanismo, el desarrollo de habilidades de investigación, la empatía entre estudiante y profesor, entre otra amplia gama de objetivos.

Adicionalmente, este estudio nos provee información muy importante para conocer el rumbo de los retos y las oportunidades para futuras capacitaciones. Igualmente, nos da luz acerca del punto actual de la cuestión y a la vez, nos abre la oportunidad para futuras investigaciones, acerca de los alcances que estas tecnologías están teniendo en el desempeño tanto de profesores como de estudiantes.

Como investigadores protagonistas dentro del contexto estudiado, nos sentimos bastante complacidos con los resultados proporcionados por este estudio. Descubrimos que la mayoría de docentes son usuarios activos de las TIC, y no sólo eso, sino que evidenciaron interés por la adaptación al uso de estas herramientas tecnológicas. El interés y el uso continuo de las TIC, por parte de los docentes, nos hace inferir 
que los estudiantes de las carreras en inglés ofrecidas por la ELCL se están viendo beneficiados.

Se evidencia que en el Área de Inglés en la ELCL existe una apropiación de las TIC para cumplir con los objetivos de los cursos donde predominan la formación pedagógica de los cursos de enseñanza y el dominio de las destrezas lingüísticas. Siendo el BEI una carrera acredita$\mathrm{da}$, esta garantiza al estudiantado y al país un programa de estudio de alta calidad por medio de mejoras sistemáticas y una de ellas es la formación académica de los docentes quienes se caracterizan por su entrega a la academia, tal y como lo indica Zabalza (2007, p. 9) "En lo que nos afecta a los profesores, se está planteando la necesidad de una más clara profesionalización de los docentes que, como cualquier otro profesional, hemos de poseer aquel conjunto de competencias docentes que nos acrediten como formadores bien formados." Así se demuestra en la tabla 1 sobre las capacitaciones recibidas en los últimos cinco años.

Entre las limitaciones que se encuentran al realizar un estudio como este percibimos la dificultad de que los docentes se tomen el tiempo para contestar cuestionarios o encuestas. En nuestro caso, la muestra fue bastante representativa; no obstante, sabemos del reto que esta tarea puede representar.

En cuanto a las recomendaciones, extraemos del estudio que aún hay camino por recorrer para alcanzar un nivel óptimo de utilización de las TIC por parte de los docentes. Aún hay una brecha en cuanto a los recursos físicos tecnológicos existentes en la ELCL y la amplia gama que existe en el mercado; a modo de ejemplo, las aulas de la ELCL no cuentan con proyectores, pantallas de proyección, pantallas interactivas y otros recursos que facilitarían el uso de las TIC. La tecnología de hoy se mueve a pasos agigantados y mantenerse a su ritmo es un gran desafío. La ELCL necesita hacer esfuerzos aún mayores en cuanto a la capacitación de sus docentes, así como en la provisión de recursos tecnológicos que faciliten el rápido acceso desde cualquier parte del campus e incluso fuera de él, con el fin de que los docentes no encuentren ningún tropiezo o desencanto en la utilización de las TIC. 


\section{Referencias bibliográficas}

Ávalos, G. G. (2008). El uso de la tecnología de la información y la comunicación y el diseño curricular. Educación (03797082), 32(1), 77-97.

Adams, S. (2014). El uso de las tecnologías en el Simposio: Impacto de las TICs en la educación 2014. Universidad Nacional, Heredia, Costa Rica. Recuperado de http://es.scribd.com/ doc/243972911/Memoria-Congreso-de-Profesinales-en-Orientacion-2014-pdf\#scribd

Beltrán,M.(febrero,2014).Menterígidavs. Menteflexible.Recuperadode http://www.encoherencia.com/mente-rigida-vs-mente-flexible/

Blanco J. (s.f.). Las TICs en la docencia universitaria Madrid, España. Universidad Politécnica Recuperado de http://www.edificacion. upm.es/ponencias/ponencias/Conferencia.pdf

Barrera, A. (2010). La capacitación y actualización docente en el uso de recursos Tecnológicos, para mejorar la práctica educativa. Recuperado de http://ece.edu.mx/ecedigital/files/ArticuloAbigail.pdf

Chaves, O., y Gutiérrez, N. (2007). Pedagogía virtual en la era tecnológica. Rhombus, (edición Vol. 3, No 10) ULACIT, San José, Costa Rica. Recuperado de http://www.ulacit.ac.cr/files/careers/67 r1002quince.pdf

Díaz Larenas, C. H., Jansson Bruce, L. E., y Neira Martínez, A. C. (2011). Percepciones de profesores y estudiantes chilenos de educación media acerca del papel de la tecnología en la clase de inglés como lengua extranjera. Revista Lasallista De Investigación, 8(2), 53-60. Recuperado de http://www.redalyc.org/articulo.oa? id $=69522607006$

Fernández, R. R., Server, P. M. y Carballo, C. E. (enero, 2006). Aprendizaje con nuevas tecnologías paradigma emergente. ¿Nuevas modalidades de aprendizaje? EDUTEC. Revista Electrónica de Tecnología Educativa, 20, 1-24. Recuperado de http://edutec.rediris.es/Revelec2/revelec20/raul20.pdf

García-Vera, Antonio. (2004). Las Nuevas tecnologías en la enseñanza. Temas para el usuario. Madrid: Akal. Impreso.

Gutiérrez, M. M. (2009). La conveniencia en el uso de la tecnología multimedia en el aula universitaria. Hospitalidad ESDAI, (16), 61-76. 
Nussbau, S., y Ritter, L. (2012). Connected Educator: Learning and leading in a digital age. Bloomington, USA: Solution Tree Press. Impreso.

Pizarro, G., y Cordero D. (2013). Las TIC: Una herramienta tecnológica para el desarrollo de las competencias lingüísticas en estudiantes universitarios de una segunda lengua. Revista Electrónica Educare, 17 (3) 277-292. Recuperado de http://www.revistas. una.ac.cr/index.php/EDUCARE/article/view/5246/5017

Ramírez, E., Cañedo, I., y Clemente, M. (2012). Las actitudes y creencias de los profesores de secundaria sobre el uso de Internet en sus clases. Comunicar, 19(38), 147-155

Turkle, S. (1997) La Vida en la Pantalla: La construcción de la identidad en la era de Internet. Barcelona, España: Piados. Impreso.

Zabalda-Beraza, M. (2007). Competencias docentes del profesorado universitario: Calidad y desarrollo profesional. Madrid: Narcea. Recuperado de http://books.google.co.cr/books?hl=en\&lr=\&i$\mathrm{d}=$ ho6AanfMHy8C\&oi $=$ fnd\&pg $=\mathrm{PA} 7 \& \mathrm{dq}=\mathrm{un}+$ plan + de + estudio + universitario\&ots $=\mathrm{NpM} 02 \mathrm{taO} 4 \mathrm{~K} \& \mathrm{sig}=\mathrm{N}-\mathrm{HoG} \_65 \mathrm{DVo}-$ 9LEXYFD0oxaKaqSE\&redir_esc $=\mathrm{y} \# \mathrm{v}=$ onepage $\& \mathrm{q}=\mathrm{un} \% 20$ plan\%20de \%20estudio\%20universitario\&f=false 


\section{Apéndice}

\section{UNIVERSIDAD NACIONAL}

Facultad de Filosofía y Letras

Escuela de Literatura y Ciencias del Lenguaje

Proyecto: Gestión de la calidad del Bachillerato en la Enseñanza del Inglés (BEI) de la Universidad Nacional, Sede Central

\section{Cuestionario: Las TICs como Herramientas pedagógicas Académicos Área de Inglés de ELCL}

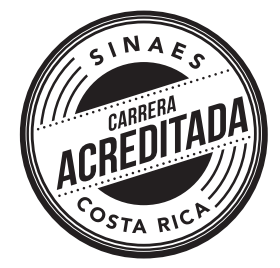

Estimados académicos del Área de Inglés de la ELCL:

Con el propósito de recolectar información acerca del uso de las TICs para enriquecer nuestra práctica docente, les solicitamos muy atentamente contestar este cuestionario basándose en su experiencia en clase.

1. ¿Cuáles de los siguientes recursos tecnológicos utiliza usted para impartir sus lecciones?

Puede elegir varias opciones.

$\square$

office

$\square$ Computadoras

Proyectores

Páginas web

$\mathrm{B} \log \mathrm{s}$

Correo electrónico
DVDs

Podcats

Chats

Prezi

Educaplay

Redes Sociales

Voice Thread 


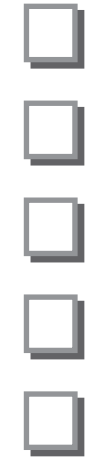

Fórums

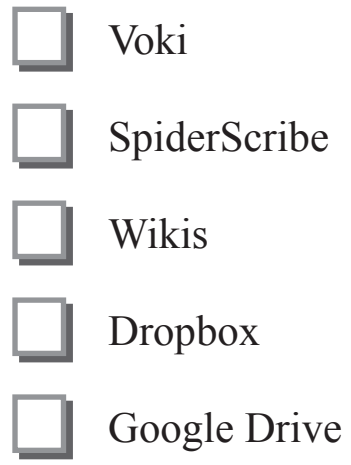

2. ¿Utiliza usted algún otro recurso tecnológico para impartir sus lecciones a parte de los mencionados anteriormente?

Mencione los otros recursos tecnológicos que usted utiliza.

3. ¿Con qué frecuencia utiliza usted las TICs en sus clases?

Piense en un curso en el que se encuentra con sus estudiantes dos veces por semana.
$\square$

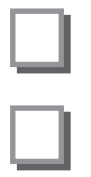
En ambas sesiones
En una sesión
2 o 3 veces al mes
$\square$ Algunas veces durante el semestre

4. ¿Utiliza usted la web para obtener recursos para el planeamiento de sus lecciones? *

Giempre que planeo mis lecciones utilizo recursos de la web.

En algunas ocasiones lo hago, tengo suficientes libros y ellos me ayudan.

Casi nunca lo hago, mis libros y mi experiencia me ayudan. 
5. ¿Por qué considera usted necesario utilizar los recursos que ofrece la web para la enseñanza del inglés? *

6. ¿Cuáles destrezas lingüísticas desarrolla/mejora mediante las herramientas tecnológicas que utiliza en sus cursos?

Por favor refiérase a la herramienta y a la destreza que busca desarrollar al utilizar esta herramienta.

7. ¿Cuáles de los siguientes aspectos busca fomentar y/o mejorar en la clase al utilizar las diferentes herramientas tecnológicas?

Puede marcar varias opciones.

$\square$ Motivación

$\square \quad$ Interacción de grupo

$\square$ Confianza para aprender la lengua

$\square \quad$ Necesidades especiales

$\square$ Autoestima

$\square$ Aprendizaje independiente

Aprendizaje del uso de nuevas herramientas tecnológicas para practicar el idioma

Desarrollo de diferente estilos de aprendizaje 
Acercamiento a la realidad que vive el estudiante

Dinamismo en clase

8. ¿Cuál otro aspecto considera usted que está fomentando al utilizar las herramientas tecnológicas?

9. ¿Recibe usted capacitación constante sobre el uso de diferentes tecnologías de información y comunicación por parte de la UNA?

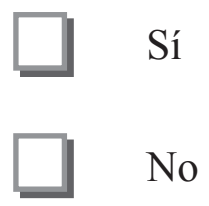

11. ¿Recibe o ha recibido usted capacitación sobre el uso de tecnologías de información y comunicación en otras instituciones académicas?

Mencione de cuáles lugares.

12. ¿En qué necesita usted capacitarse referente a las TICs?

Por favor anote las tecnologías de información y comunicación que usted considere necesarias para futuras capacitaciones en la ELCL. 